\title{
Die Post-Truth-Ära: Kommunikation im Zeitalter von gefühlten Wahrheiten und Alternativen Fakten
}

Ralf Hohlfeld

Alexa und Siri, die Cloud und dein Boss Die stille Post und die Stimmen in deinem Kopf Der Guru, die Trainer, der TÜV und der Mob Der hat's von Tinder und die haben's von Gott Deichkind: »Wer sagt denn das?«

Abstract

In diesem Beitrag wird argumentiert, dass das sogenannte postfaktische Zeitalter sich von den herkömmlichen Praktiken der Lüge und Vertuschung in der politischen Kommunikation deutlich unterscheidet und mittels neuer Narrative eine neue, fragwürdige Qualität in der öffentlichen Kommunikation etabliert wird: Wahrheit und Lüge werden rhetorisch verwechselbar gemacht, ihre Wertigkeit eingeebnet. Die dazu eingesetzten Instrumente und rhetorischen Figuren wie Alternative Fakten und subjektive Wahrheiten gehören weder in den Bereich der Missverständnisse des Alltäglichen, noch sind sie sprachliche Ausrutscher unbeholfener Krisenkommunikatoren. Sie sind vielmehr Schleifwerkzeuge, die von Populisten und antidemokratischen Akteuren bewusst an den demokratischen Diskurs angelegt werden und die ehernen Prinzipien der Aufklärung unterhöhlen sollen. Die Diskreditierung von Faktizität setzt bewusst bei deren Referenzsystemen Journalismus und Wissenschaft an, die für das Liefern gesellschaftlicher Wahrheits- und Wirklichkeitsentwürfe zuständig sind. Hier kommt das Demokratie-Paradoxon zum Tragen: Demokratie und die offene Gesellschaft unterliegen der grundsätzlichen Gefahr, an ihren Stärken - den von ihnen gewährten Freiheitsrechten - in paradoxer Weise zu scheitern. So muss die demokratische Gesellschaft vermehrt propagandistische Angriffe und evidenzfreie Behauptungen gegen die Institutionen von Wissenschaft und Medien aushalten, die sie qua Meinungs-, Informations- und Wissenschaftsfreiheit auch ihren erklärten Gegnern einräumt. 
In ihrem Song »Wer sagt denn das?« spielt die deutsche Hip-Hop- und Tech-Rap-Band Deichkind mit ehernen Gewissheiten des Alltags und persifliert die Vernetzung von allem mit jedem, indem sie Behauptungen, Befunde, Lebensweisheiten, Parolen und gesicherte Erkenntnisse textlich wild durcheinanderwirbelt. Zweifel daran, »dass Jugendliche keine Zeitung lesen « wechseln sich ab mit Bedenken, »das mit dem Kontrabass wären drei Chinesen« - stets abgebunden mit dem Refrain »Wer sagt denn das?«. Das Lied wurde nach Erscheinen im Sommer 2019 in der Musikszene als kritische Auseinandersetzung mit postfaktischer Meinungsbildung gefeiert (Siegmund, 2019). Die Hymne auf die Verirrungen und Verwirrungen im digitalen Informationszeitalter gipfelt dann in einem lakonischen »Ich glaub' ab jetzt nur das, was stimmt «.

Spätestens mit der Inauguration Donald Trumps am 20. Januar 2017 begann in der Öffentlichkeit das sogenannte postfaktische Zeitalter. Zwar zeichnete es sich in ersten Umrissen schon in den Neunziger Jahren des 20. Jahrhunderts ab: Ralph Keyes (2004) hatte für seine Aufarbeitung der Lügengeschichten, mit denen in den USA und Großbritannien der Irakkrieg politisch gerechtfertigt worden war, den Titel »The Post-Truth Era. Dishonesty and Deception in Contemporary Life« (Keyes, 2004) gefunden. Aber erst das auf Sean Spicers offenkundige Falschbehauptung, Trumps Amtseinsetzung hätten mehr Menschen beigewohnt als jeder anderen Amtseinführung eines Präsidenten, zurückgehende Bonmot der Beraterin Kellyanne Conway, die Regierung verfüge über so genannte Alternative Fakten (Haberkorn, 2018), brachte das allmählich heraufziehende Zeitalter gefühlter Wahrheiten sprachlich auf den Punkt: »Period«, sprach Trumps damaliger Pressesprecher.

Die mit großem Selbstbewusstsein von Trumps Beraterstab hervorgebrachte Formel der Alternativen Fakten war in der westlichen Welt das erste öffentliche politische Bekenntnis zur Postfaktizität und zugleich die Bekräftigung des Willens, mit solchen rhetorisierten politischen Kampfbegriffen die kommunikative Auseinandersetzung um die Deutungshoheit im digitalen Zeitalter zu führen. Anders als im Falle von Unehrlichkeit, Lüge und Unwahrheit in der politischen Kommunikation, die auf eine lange Tradition zurückblicken können, soll hier aber nichts vertuscht werden. Das Abstreiten der faktischen Wahrheit wird mit strategischem Vorsatz zum Programm erhoben. Es ist daher an der Zeit, mit dem Märchen aufzuräumen, Postfaktizität sei alter Wein in neuen Schläuchen. Es geht in der Post-Truth-Ära nicht um die anthropologische Konstante, Erfolge in der Politik durch Unwahrheiten zu erzielen und sich mit Lügen aus der politischen Verantwortung zu stehlen. Es geht um eine neue Qualität in der öffentlichen Kom- 
munikation: Wahrheit und Lüge seien verwechselbar und hätten denselben Wert.

\section{Der Geltungsanspruch von Fakten}

Alternative Fakten sind seit dem Inaugurationsdiskurs als geflügeltes Wort gebräuchlich und werden allgemein als Versuch eingeschätzt, ein neues Narrativ zu etablieren (Schwan, 2017). Es wird durch diese rhetorische Rahmung vorgegeben, dass Fakten und Alternative Fakten prinzipiell denselben Wert in der öffentlichen Kommunikation und im politischen Diskurs beanspruchen können. Der neue Frame Alternative Fakten eröffnet nun sprachlich die Möglichkeit, nicht nur über die Selektion, Interpretation und Gewichtung von Fakten zu streiten, sondern die Fakten, die zwar das Ergebnis von Selektionen (und damit konstruktivistisch gesprochen Beobachtungen) sind, selbst zur Disposition zu stellen. Wahr oder falsch wäre demnach keine Frage, die durch Fakten verifizierbar ist, da die Fakten schon als Alternativen formulierbar und damit die »Tatsachenwahrheiten« (Arendt, 2013) bestreitbar sind.

Damit wird zugleich die Frage aufgeworfen, was Fakten im heutigen Verständnis eigentlich sind - und was sie sein sollen. Sind es in unser aller Verständnis (noch) unumstößliche, letztendliche Wahrheiten in einem alteuropäischen Sinne der Aufklärung - also Tatsachen, Sachverhalte oder so genannte Tatsachenwahrheiten, die Hannah Arendt (2013) von Deutungen, Meinungen und ethischen Prinzipien abgrenzt? Denn »Wahrheit wird gemeinhin zu jenen letzten begrifflichen Bastionen gezählt, die nicht individualisierbar, besitzfähig oder gar konsumierbar sein sollen oder dürfen«, wie Meredith Haaf $(2020,4)$ schreibt. Oder sind es dem Wortsinn des lateinischen Ursprungs factum nach menschengemachte Zuschreibungen? Diese an den Konstruktivismus angelehnte Sichtweise würde die Aussage des ehemaligen Pressesprechers Donald Trumps mit dem Hinweis legitimieren, dass es unterschiedliche Modelle von Wirklichkeitskonstruktionen gibt, die schon auf der Tatsachenebene kognitiv variierbar sind. Geht es also um die Vorherrschaft eines bestimmten erkenntnistheoretischen Paradigmas?

Wenn man mit Arendt (2013) argumentiert, dass Tatsachenwahrheiten Deutungen und Meinungen überlegen sind, weil durch sie die Erkenntnis über die Welt belegbar ist und eine von Menschen bezeugbare Realität entsteht, die der Wahrhaftigkeit verpflichtet ist, dann sind Alternative Fakten nicht mehr als ein Oxymoron - eine widersprüchliche Wortverbindung mit 
dem Ziel der Provokation. Zumindest unter der Voraussetzung, dass man wie Sean Spicer behauptet, man könne bisweilen uneins mit den Fakten sein: »Sometimes, we can disagree with the facts « (zitiert in Hendricks \& Vestergaard 2018, 94). Ob am Tage der Inauguration des Präsidenten die Sonne über Washington schien, ob die Anzahl der physisch anwesenden Zuschauer größer war als je zuvor, ob die Transportunternehmen 500.000 Fahrten oder eine Million Fahrten an jenem Tage zu organisieren hatten, ist jedoch keine Frage der Bewertung oder Deutung. Das sind Tatsachenwahrheiten, die im Verständnis von Hannah Arendt (2013) nicht hintergehbar sind. »Natürlich kann man bestreiten, dass es tatsächlich Fakten sind. Aber mit Fakten uneinig zu sein, bedeutet mit der Wirklichkeit uneinig zu sein« (Hendricks \& Vestergaard, 2017, 94, Hervorhebung im Original).

In diesem Sinn kann man nicht mit Fakten uneins sein, höchstens mit ihrem Geltungsanspruch. Man kann dann folglich im Prozess des kommunikativen Handelns gleich alle vier der von Jürgen Habermas (1981) formulierten Geltungsansprüche an kommunikative Verständigung durch Diskurs - Wahrheit, Wahrhaftigkeit, Richtigkeit und Verständlichkeit - auf einmal bestreiten; mit vorhersehbaren Folgen für die Verständigung. Dann bedeutet Faktenleugnung eine bewusste Absage an den Hauptzweck von Kommunikation, nämlich Verständigung, denn »über eine Behauptung, für die ein Faktencheck rundherum abgelehnt wird, indem man von , alternativen“ - nicht von anderen! - Fakten spricht, kann man sich in der Tat nicht verständigen « (Schwan 2017, 42). Denn Fakten werden in der offenen Gesellschaft in einem Verständigungsprozess etabliert, der auf Erfahrungen, nicht auf Eindrücken und Wünschen basiert (Strasser, 2017).

Alternative Tatsachen sind ein Oxymoron, aber worum geht es dabei? Es geht zum einen um die banale Feststellung, dass in der Ära der Postfaktizität im Alltäglichen der Umgang mit Fakten zunehmend in den Bereich der Geschmacksfragen zu fallen scheint (Haaf, 2020) und sich daran die Frage anschließt, was bedeutet Wahrheit im öffentlichen Leben noch? Gerade wenn es um Herausforderungen des Alltags geht, wenn bei der Suche nach Gesundheit die Schulmedizin auf eine Komplementärmedizin wie die Homöopathie trifft. Wenn sich klinische Evidenz mit persönlicher Erfahrung messen muss, aus der im Diskurs über die Wirksamkeit von Therapien der Topos »subjektive Wahrheit« filtriert wird. Hier prallen zwei Zugänge zur Wirklichkeit aufeinander. Die Schulmedizin unterliegt einem faktenbasierten Prüfsystem. Faktisch bedeutet überprüfbar: Es handelt sich um einen Sachverhalt, die Therapieform, der sich einer Prüfung unterziehen lässt, unbesehen des Maßstabs, der für die Prüfung angelegt wird. Eine subjektive oder eine gefühlte Wahrheit, die dem Muster der Postfaktizität folgt, sieht 
dagegen keine Notwendigkeit für eine evidenzbasierte statistische Prüfung; eine Behauptung, die auf affektive Zustimmung eines Subjekts gerichtet ist, reicht aus. Mithin ist die gefühlte Wahrheit die kleine Schwester der Plausibilität: Es reicht, wenn etwas so sein könnte, wie es behauptet wird, weil ein solcher Sachverhalt, eine Heilung oder Wunderheilung, in anderen Kontexten so oder so ähnlich schon vorgekommen oder zumindest gewünscht worden ist. Subjektive Wahrheit ist auch eine Umschreibung für (die unbotmäßige Inanspruchnahme von): Ansichtssache.

Zum postfaktischen Handeln im Alltag, das auf dem Primat des Geschmacks und der Ästhetik gründet, gehört freilich auch der laxe Umgang mit der Wahrheit im Journalismus. Auch bei der seit den Neunzigerjahren zu beobachtenden Konvergenz von Fakten und Fiktionen, die im Boulevardjournalismus und im Infotainment-Format Gestalt gewannen und im »Faction-Journalismus« (Weber, 2002, 14) der journalistischen Fälscher Michael Born und Claas Relotius besonders stark ausgeprägt sind, spielt der Bezug auf Plausibilität eine große Rolle: Wahr ist etwas, wenn es so oder ähnlich hätte sein können. Die dahinterliegenden Konstruktionsprinzipien wurden schon in der Programmatik des literarischen Journalismus aufgerufen, der den Zeitgeistjournalismus der Achtzigerjahre und den früheren New Journalism eines Tom Wolfe antrieb. Der Interview-Fälscher Tom Kummer hatte diesen »Boderline-Journalismus« seinerzeit in einem Gespräch mit dem Spiegel die »Implosion des Realen« genannt und später in dem Buch »Blow up. Eine wahre Geschichte im Zeitalter des Fakes « (Kummer, 2007) nachträglich eine infame Medientheorie daraus gefertigt.

Zum anderen aber geht es beim Narrativ der Alternativen Fakten um das Unterhöhlen eherner Prinzipien der Aufklärung. Deuten ersetzt Erkennen, Gerüchte treten an die Stelle von Gewissheiten, das Hörensagen desavouiert die Recherche. Und weiter: Behauptung vor Evidenz, Postulat vor Begründung, das Einpassen ungeprüfter Informationen in die eigene Vorstellungswelt statt der Argumentation. Mit dem Prinzip der sinnesbasierten Evidenz herrschte ja schon seit der Aufklärung die Vorstellung vor, dass in der modernen Gesellschaft zwischen Wahrheit und möglicher Lüge unterschieden werden kann, selbst wenn politische Propaganda die Menschheit gelehrt hat, dass damit keine Immunisierung gegen den Totalitarismus verbunden ist.

Insofern sind Alternative Fakten ein krudes politisches Paradigma, das die gefühlten Wahrheiten des postfaktischen Zeitalters bis auf die Ebene der Tatsachen herunterbrechen und auf der Faktenebene die Anerkennung einer möglichen Kontradiktion erzwingen will, die das Gefühl über die Tatsache stellt und subjektive Wahrheiten über die Empirie triumphieren lassen will. 
Man kann auf diese Weise dann den Brexit als Freiheitsversprechen und Wachstumsmotor verkaufen, obwohl die Kosten den Nutzen volkswirtschaftlich weit übersteigen. Man kann die Presse zum Feind des Volkes erklären, obwohl diese dem Gemeinwohl verpflichtet ist und eine öffentliche Aufgabe wahrnimmt, abgeleitet aus der Verfassung. Man kann Philanthropen zu Terroristen erklären, Klimaaktivisten zu Agenten dunkler Mächte und so weiter. In diesem Lichte besehen, gehören Alternative Fakten nicht in den Bereich der Missverständnisse des Alltäglichen. Sie sind auch kein sprachlicher Ausrutscher unbeholfener Krisenkommunikatoren, sondern ein Schleifwerkzeug, das bewusst an den demokratischen Diskurs angelegt wird.

\section{Postfaktizität: Die Karriere eines Begriffs}

Das Ringen über die faktische Deutungshoheit bezüglich der Rahmenbedingungen von Donald Trumps Amtsantritt dürfte die neue Qualität eines postfaktischen Zeitalters offenkundig gemacht haben, die amtliche Auszeichnung des Begriffs liegt jedoch zeitlich davor. Schon Ende 2016 haben sowohl das Oxford English Dictionary als auch die Gesellschaft für deutsche Sprache in Wiesbaden die Begriffe post-truth und postfaktisch in Großbritannien und Deutschland jeweils zum Wort des Jahres erklärt. Vermutlich hatte die Wahl Trumps Anfang November dazu beigetragen, dass sich der Begriff post-truth in Großbritannien gegen die Wortkonkurrenten brexiteer und chatbot durchgesetzt hat, in Deutschland dürfte eine Bemerkung der Kanzlerin Angela Merkel eine Rolle gespielt haben, die sie Mitte September 2016 nach schlechten Wahlergebnissen in Berlin und Mecklenburg-Vorpommern gemacht hatte: »Es heißt ja neuerdings, wir lebten in postfaktischen Zeiten« (Breuer, 2016), vier Jahre später fordert sie auf dem Weltwirtschaftsforum in Davos: »Wir müssen Emotionen mit den Fakten versöhnen« (Lehmann, 2020).

Es bedurfte also nur noch der Erweiterung und Konkretisierung von Postfaktizität zum begrifflichen Erscheinen Alternativer Fakten durch die Trump-Entourage, um eine Breitenwirkung zu entfalten, die weit über kalendarische Auszeichnungen aus dem Elfenbeinturm hinausreicht. Die Kommunikation des US-Präsidenten Trump spitzte eine Rhetorik zu, die in der politischen Kommunikation der vergangenen fünf Jahre sukzessive Formen angenommen hatte. Die Erosion von Fakten zugunsten von Gefühlen, von Empirie zugunsten Subjektivität, lag gleichsam in der Luft. 
Die neuen Narrative Post-Truth, Postfaktizität, gefühlte Wahrheiten, subjektive Wahrheiten und Alternative Fakten besetzen gemeinsam einen semantischen Raum, der sich allmählich unter den wichtigen politischen Entscheidungen der jüngeren Vergangenheit verdichtet hat. Dieser Raum überwölbt die vielen Arten der Desinformation, mit denen heutzutage in fast selbstverständlicher Weise Politik gemacht wird. Die Destabilisierungsaktivitäten in den postsozialistischen Staaten der ehemaligen Sowjetunion und anderen Staaten Osteuropas, der asymmetrische Krieg zwischen Russland und der Ukraine im Cyberspace, die Brexit-Kampagne samt Geiselnahme der EU im Austrittsverfahren, die in vielfacher Weise manipulierte Präsidentschaftswahl in den USA: Sie alle sind von diesem neuen dominanten Paradigma der strategischen politischen Kommunikation beeinflusst worden - und gleichzeitig haben diese Vorgänge von weltpolitischer Bedeutung dazu beigetragen, dass dieses Muster sich schrittweise weiter etablieren konnte.

Freilich liegt der Beginn politischer Kommunikationsbestrebungen, die Faktizität zu überwinden, deutlich länger zurück. Die Ursprünge liegen weltweit als lose Fäden in Ost und West, sie streuen im Okzident und Orient breit durch die Geschichte der Menschheit. Häufig genannt wird in diesem Zusammenhang das Bonmot von Aischylos »Im Krieg ist die Wahrheit das erste Opfer«; letztlich sind Kriege und kriegerische Auseinandersetzungen ohne Propagandalügen kaum vorstellbar. Statt an dieser Stelle eine erschöpfende weltweite Spurensuche postfaktischer Vorgänge und ihrer sprachlichen Definitionen und analytischen Bestimmungen vornehmen zu können, sei zumindest für die sprachliche Genealogie in den Vereinigten Staaten der Hinweis auf Steve Tesich gegeben, der unter dem Einfluss des Golfkriegs schon 1992 in einem Aufsatz in The Nation das Bild einer postfaktischen Nation skizzierte, das stellvertretend für die selbstreflexive Beschäftigung mit der Angst vor der Wahrheit stehen kann, die uns Menschen im Angesicht von Krieg und Krisen befällt und oftmals zu einem kollektiven Vogel Strauß-Verhalten führt: »We came to equate truth with bad news and we didn't want bad news anymore, no matter how true or vital to our health as a nation. We looked to our government to protect us from the truth « (Tesich, 1992, 12). Tesich argumentiert, dass die US-amerikanische Gesellschaft angesichts all der offensichtlichen politischen Vertuschungsversuche in der Zeit von der Nixon-Ära bis zum Golfkrieg unter der Administration von George Bush Senior die bewusste Entscheidung getroffen hatte, auf Kosten der Demokratie in einer postfaktischen Welt zu leben: »In a very fundamental way we, as a free people, have freely decided that we want to live in some post-truth world« (Tesich, 1992, 13). 


\section{Postfaktische Narrative und das Demokratie-Paradoxon}

Aus dieser Einschätzung spricht eine tiefe Enttäuschung über die Zivilgesellschaft und ihre lethargische Haltung. Doch Tesichs Diagnose aus dem Vor-Internet-Zeitalter ging nicht von einem grundlegenden Systemversagen aus, das auf einem konstitutionellen Konstruktionsfehler der Öffentlichkeit basiert. Rund drei Jahrzehnte später ist nun aus der Momentaufnahme einer postfaktischen Welt ein postfaktisches Zeitalter geworden, eine Epoche, die weder ein historischer Unfall noch ein evolutionärer Zufall ist. Die Narrative der sogenannten gefühlten Wahrheiten, subjektiven Wahrheiten und Alternativen Fakten, sind wie auch die Kampfbegriffe »Fake News Media«, Mainstream-Medien und Lügenpresse zu etablierten Werkzeugen der strategischen politischen Kommunikation geworden. Ihr Zweck: Demokratiezersetzung. Ihr instrumenteller Einsatz richtet sich unverblümt gegen die offene Gesellschaft und ihre liberalen Werte. Dahinter stecken Anhänger autoritativer Herrschaftsformen, Nationalisten, xenophobe Rechtsextreme und Rechtspopulisten, die die offene Gesellschaft anfeinden.

Die von ihnen genutzten Narrative des postfaktischen Zeitalters wirken gleichsam als Auslöser des latenten Demokratie-Paradoxon: Die Stärken der Demokratien, nämlich die von ihnen konstitutionell gewährten Freiheiten, sind zugleich ihre Schwächen. So wie der Rechtsstaat damit leben muss, dass mit den Grundsätzen des positiven Rechts nicht immer Gerechtigkeit geschaffen wird und sich Recht auch gegen den Rechthabenden richten kann, unterliegen Demokratie und die offene Gesellschaft der grundsätzlichen Gefahr, an den von ihnen gewährten Freiheitsrechten in paradoxer Weise zugrunde zu gehen. Denn die demokratische Gesellschaft muss propagandistische Angriffe, evidenzfreie Behauptungen gegen das so genannte Establishment und gegen sie gerichtete Hetzattacken aushalten, da sie sie qua Meinungs-, Kunst- und Wissenschaftsfreiheit ihren erklärten Gegnern einräumt. Eine liberale Demokratie mit offener, pluralistischer Gesellschaft lebt immer mit den inhärenten Bedrohungen der missbräuchlichen Nutzung der von ihr gewährten Freiheit. Nur, dass im zurückliegenden analogen Zeitalter die Breitenwirkung für den systemischen Missbrauch noch nicht gegeben war.

Die Demokratie konnte die missbräuchliche Nutzung der von ihr gewährten Freiheiten aushalten, solange es einen Konsens darüber gab, dass die Institution Journalismus durch faktenbasierte Recherche Kritik und Kontrolle ausübt und es möglich war, die politische Lüge nicht nur zu entlarven, sondern mit der Entlarvung den Lügner auch bloßzustellen. Seit der 
digitalen Wende zeichnet sich jedoch ab, dass das professionelle Informationssystem Journalismus sein Monopol bei der Herstellung von Öffentlichkeit verloren hat und sich nun vermehrt so genannten alternativen Medien gegenübersieht, die an keine öffentliche Aufgabe gebunden sind und sich wie Breitbart News in den USA oder Compact in Deutschland der liberalen Demokratie nicht verpflichtet fühlen.

Da aber Demokratie auf der Idee des Marktplatzes der Meinungen sowie des Wettbewerbs der besten Ideen und Argumente aufbaut, hängt sie von geprüften und prüfbaren Informationen ab. Die Lüge und das Bestreiten von Tatsachenwahrheiten unterhöhlen den rationalen Diskurs, indem sie dessen Geltungsansprüche ignorieren. Auf diese Weise führen sie zur Vermachtung und Okkupation des Meinungswettstreits. Dieser ist nämlich auf Fakten angewiesen; der evidenzbasierte Meinungsaustausch ist im demokratischen Willensbildungsprozess konstitutiv. Wenn es sich aber im Informationssystem der Gesellschaft durchsetzt, dass gefühlte Wahrheiten dieselbe Beweis- und Argumentationskraft erhalten wie Tatsachenwahrheiten, wenn also Meinungen nicht mehr auf Fakten basieren, sondern - da man Fakten nun bestreiten kann - auf Meinungen über Meinungen, dann ist das eine gravierende Gefährdung der Vorstellung, dass in der Demokratie Entscheidungen auf guten Informationen gründen. Oder pointierter: Wenn man Fakten nicht akzeptiert, dann hat Kontrolle keinen Sinn mehr, dann ist das Prinzip von Checks and Balances obsolet.

Evelyn Roll (2016) nimmt ihre Zunft in die Pflicht, wenn sie schreibt: $»$ Vielleicht müssen wir Journalisten neu lernen, dass man einen Text durchaus auch mal beginnen kann mit den drei Wörtern: Das ist falsch.« Über das Leugnen des Klimawandels oder die Evolution oder über Hetze gegen Minderheiten könne man nur schreiben, wenn man die dahinterstehenden Erfindungen auch Erfindungen nenne: »Jeder hat das Recht auf eine eigene Meinung, aber niemand hat das Recht auf eigene Fakten. Es wird überlebenswichtig sein für die Demokratie, eine Lüge wieder eine Lüge zu nennen. Wenn jemand behauptet, die Erde ist eine Scheibe, darf die Schlagzeile eben nicht sein: ,Streit über die Form der Erde“"« (Roll, 2016).

\section{Wissenschaft und Journalismus unter Druck}

Unter dem Blickwinkel der demokratietheoretischen Besorgnis ist es umso bitterer, dass die Diskreditierung von Fakten und Faktizität bei deren Referenzsystemen Journalismus und Wissenschaft ansetzt: zwei soziale Systemen, die eine Zuständigkeit für das Liefern von gesellschaftlichen Wahr- 
heits- und Wirklichkeitsentwürfen für sich reklamieren. In gewisser Weise arbeiten beide Systeme empirisch, das eine eher auf der Basis von Fallbeispielen und Recherchen von Indizien zur Verallgemeinerung, das andere streng auf der Basis von Standardisierung und Reproduzierbarkeit. Bei ähnlichem Anspruch - Suche nach Wahrheit und Wirklichkeitsentwürfen operieren beide Bereiche tatsachenorientiert, wenn auch auf unterschiedliche Weise, mit verschiedenen Strategien, Methoden und Heuristiken. Wissenschaftliches Wissen ist prinzipiell evidenzbasiert und damit wahrheitsfähig, es ist nicht erst seit Karl Raimund Poppers Logik der Forschung $(1934 / 1989)$ der Falsifikation ausgesetzt - anders als das bloße Meinen und Behaupten. Das Ersetzen einer älteren Theorie durch eine neue, die Verfeinerung der Methoden und die Falsifikation von deduktiven Hypothesen sind Mittel der Wahrheitsfindung. Auch der Journalismus teilt die Auffassung, dass die Erkenntnis von heute der Irrtum von morgen ist. Den vermeintlichen Objektivitätsanspruch versuchen Journalistinnen und Journalisten durch vorurteilsfreie, offene und dokumentierte Recherche sowie intersubjektive und transparente Darstellung einzulösen - zumindest in theoretischer Hinsicht und, was den normativen Anspruch betrifft.

Herausgefordert werden beide Systeme durch dreierlei: die Digitalisierung der öffentlichen Kommunikation, die Krise der westlichen Demokratien und die mannigfachen Verschränkungen beider Entwicklungen. Der Nachrichtenjournalismus krankt daran, dass soziale Netzwerke mit dem Liken, Empfehlen und Teilen Metriken entwickelt haben, die - aufmerksamkeitsökonomisch betrachtet - die Nüchternheit und Sachbezogenheit des auf Themen von sozialer Verbindlichkeit abzielenden Nachrichtenjournalismus strukturell benachteiligen. Die daraus entstehenden vielbesungenen Filterblasen und Echokammern des Internets führen schrittweise zur Kultur einer emotional aufgeladenen Behauptungs- und Empörungskommunikation, die sich immer wieder in Beschimpfungen demokratischer Institutionen, des so genannten Establishments und vorgeblicher Eliten entlädt - gemeint ist die Trias aus Politik, Medien und Wissenschaft. Die alternativen Medien der antidemokratischen Propagandisten bedienen sich der algorithmischen Vorteile, die Hass, Hetze und Lügen gegenüber der sachbezogenen Darstellung politischer Themen besitzen und spielen diese im Konzert mit den sozialen Netzwerken gnadenlos aus: »Neue Propaganda- und GefühlsMedien werden zu Pseudojournalismus aufgeblasen, der ansagt, was ,das Volk' angeblich will« (Roll, 2016).

Wissenschaft und Journalismus stecken in einer Falle, die zum einen dadurch gestellt ist, dass affektive und emotive Inhalte es leichter haben, auf den Verweisstrukturen der sozialen Netzwerke in den Aufmerk- 
samkeitsfokus der Nutzer vorzustoßen, und zum anderen darin besteht, dass mit Multiplikation von Information und der Akzeleration von Kommunikation eine Komplexität entstanden ist, die nicht mehr ohne weiteres bearbeitbar ist. Die Entwicklung des Internets und seiner sozialen Medien hat zwar die Informationsangebote weltweit in unfassbarer Weise vergrößert, die Auffindbarkeit von Wissensbeständen und geprüften Informationen aus beiden Systemen ist für die Nutzerinnen und Nutzer indes schwieriger geworden. Neben den Aufmerksamkeitsmetriken der sozialen Medien sind dafür die Zugangskosten zu Informationen und zum Wissen über deren Qualität verantwortlich. Denn nicht nur die Generierung und Prüfung von Informationen durch Recherche und Gegenrecherche im Journalismus und durch aufwändige Prüf- und Reflexionsverfahren wie das Peer-Reviewing in der Wissenschaft verursachen enorm hohe Kosten, sondern auch die Rezeption. Denn während für exklusiven Premiumjournalismus der New York Times oder der Süddeutschen Zeitung heute viel Geld verlangt wird und wissenschaftliche $A$-Journals mit hohem Impact-Faktor kaum jemals im $O$ pen-Access-Modus zugänglich sind, können im Internet auf der anderen Seite jegliche Behauptungen und Thesen quasi ohne Zugangsbarrieren in einem Tweet, einem Posting, einem Blogeintrag oder einem YouTube-Video publiziert und genauso einfach kostenfrei rezipiert werden. Der Politiker Trump hat dieses Prinzip früh erkannt und dessen Wirkungsmächtigkeit verstanden.

Sichtbar war der Einfluss der digitalen Aufmerksamkeitsmetriken lange Zeit auch bei den Eintragungen in Suchmaschinen, etwa im Falle von Verschwörungsmythen: Klimawandel, Chemtrails, Reptiloide, Flacherdtheorie, Marslandung - oft stehen die nicht evidenzbasierten Beiträge oben in den Suchlisten und erwecken auch bei neutralem Suchinteresse den Eindruck, dass ihnen wegen der Priorisierung durch vermeintlich neutrale Algorithmen mehr Gewicht zukommt. Erschwerend kommt hinzu, dass die wissenschaftsjournalistische Verarbeitung komplexer wissenschaftlicher Erkenntnisprozesse oft nicht das wünschenswerte Niveau erreicht. So entsteht durch unzureichende Vermittlung von Studienergebnissen zu Umwelt- und Gesundheitsthemen für die Rezipienten das Bild widersprüchlicher Befunde, welches die Menschen weiter verwirrt und in ihrer Haltung bestätigt, dass wissenschaftliches Wissen für die Lösung von alltäglichen Problemen keinen bedeutsamen Wert besitzt.

Die Post-Truth-Ära wird daher auch als Vorbote gedeutet, der das Ende des Zeitalters der Wissenschaft einläuten könnte. So hatte sich mit der Leugnung der Evolution durch den Kreationismus schon über Jahrzehnte in große Teile der Gesellschaft eine Wissenschaftsskepsis gefräst, die nun 
durch das Bestreiten des Klimawandels, die Verharmlosung von Feinstaubund Dieselemissionen sowie extraterrestrische Verschwörungserzählungen weiter gefüttert wird. Der hier wirksame wissenschaftsbezogene Populismus spricht den etablierten wissenschaftlichen Institutionen die Deutungsund Entscheidungshoheit gegenüber den gesellschaftlichen Wissens- und Machtansprüchen ab und setzt an die Stelle der akademischen Eliten das Volk, dessen gesunden Menschenverstand und andere Alltagsheuristiken »wäre die Erde eine Kugel, warum ist dann der Horizont nicht krumm?«.

Auch der Faktizitäts- beziehungsweise Wirklichkeitsanspruch des Journalismus gilt - seit die Formeln Lügenpresse und »Fake News Media« den digitalen und den analogen Raum erobert haben - als derart unterminiert, dass er vor allem im digitalen Raum mühelos durch das Prinzip des kostenfreien Behauptens und Proklamierens ausgehöhlt werden kann.

An beiden Systemen lässt sich das Demokratieparadoxon insofern besonders gut sichtbar machen, dass beide als Grundpfeiler der Demokratie mit ihren Privilegien im Range eines Grundrechts, und zwar der zweckungebundenen Wissenschaftsfreiheit und der Meinungs- und Informationsfreiheit, letztlich Komplexität produziert und Kontingenz hervorgebracht haben. Diese führt nun mittels digitaler Vervielfältigung und missbräuchlicher Interpretation durch Laien zu vermehrter kognitiver Überlastung der Menschen, Bürger, Nutzer. Im Ergebnis muss die demokratische Gesellschaft infolge unzureichender Kapazitäten der Komplexitätsbearbeitung zahlreicher Bürger deren Meinungen aushalten, die meist keiner nachvollziehbaren Ratio folgen; Meinungen, die oft in Form scharfer Kritik an wissenschaftlichen und journalistischen Institutionen und Methoden gleichzeitig die Grundlagen der demokratischen Herrschafts- und Gesellschaftsform schleifen.

Stellvertretend für eine ganze Reihe von Fällen, in denen wissenschaftliche Institutionen und Methoden durch verschwörungstheoretische Kommunikation in Misskredit geraten sind, sei die Kontroverse um \#Gamergate genannt. Der Streit um die Deutungshoheit im Bereich der Spielekritik in der Computerspiel-Branche hatte 2014 Züge eines Kulturkampfes angenommen. Nachdem feministische Spieleentwicklerinnen, durch Crowdfunding-Aktivitäten unterstützt, damit begannen, Computerspiele aus feministischer Sicht zu analysieren und in diesem Zuge schematische und sexistische Darstellungen von Frauenfiguren kritisierten, wurden diese von der Gamegate-Bewegung genauso verbal verunglimpft wie die feministischen Forscherinnen, die den daraus entstandenen Diskurs untersuchten (Chess \& Shaw, 2015). Daraus entwickelte sich eine weitere Verschwörungstheorie, die der Wissenschaft unterstellte, mit einseitigen Forschungsprogrammen 
progressive Gesellschaftsentwürfe wie den Feminismus zu fördern. Zur Konsequenz, wissenschaftlichen Verfahrensweisen jegliche Legitimität abzusprechen, ist es dann nur noch ein Katzensprung. Ähnlich erging es in der Hochphase der Corona-Pandemie der Virologie und Epidemiologie, denen von Verschwörungsgläubigen Eigennutz und der Versuch, die Gesellschaft kontrollieren zu wollen, unterstellt wurde.

\section{Das Postfaktische zieht das Overton-Fenster auf}

Dass die postmoderne Gesellschaft auch gleichzeitig eine postfaktische Gesellschaft ist, muss kein Automatismus sein. Eine solche Zwangsläufigkeit gibt es nur in technodeterministischen Vorstellungen des Sozialen. Postmoderne demokratische Gesellschaften besitzen die Freiheiten und die Freiheitsgrade, das gesellschaftlich Wünschenswerte durch Umprogrammierung dysfunktionaler Entwicklungen zu erreichen. Folgt man der Einschätzung, dass die Post-Truth-Ära zwar technische und systembedingte Ursachen hat, aber letztlich das Ergebnis institutionalisierter Handlungsstrategien ist, die auf eine Veränderung der Gesellschafts- und Staatsform drängen (durch die Überwindung der Herrschaft des Rechts und der Wissenschaft samt ihren Institutionen), dann können sich diese auch strategisch gegen eine solche Gängelung wehren.

Was ist das übergeordnete Ziel der Kommunikatoren, die an der Faktizität nagen? Was beabsichtigen die Erfinder und Entwickler des Narrativs einer relativen Wahrheit? Wie beschrieben, versuchen Demokratiegegner und Feinde der offenen Gesellschaft mit der Absage an die Hoheit evidenzbasierter Faktizität den rationalen Diskurs zu verunmöglichen, sie beabsichtigen mit der Verschleierung durch Desinformation Verunsicherung hervorzurufen und damit eine gesellschaftliche Destabilisierung zu erzwingen. Das Mittel zum Zweck ist, das Unsagbare zum Sagbaren zu erheben, Schranken zu beseitigen, die als diskursive Normen die Spielregeln in der demokratischen Öffentlichkeit repräsentieren. Sie bedienen sich dazu vielerlei Methoden, von denen die wirkungsvollste diejenige sein dürfte, mit gezielten Tabubrüchen das so genannte »Overton-Fenster" (Jacobsen, 2018) zu weiten. Der Tabubruch ist dabei nicht die simple Lüge, sondern die viel wirksamere Diskreditierung der Wahrheit. Das Overton-Fenster bildet traditionell den politisch zulässigen Handlungsrahmen beziehungsweise Kommunikationsrahmen zu einem Thema ab. Das konsentierte Spektrum der politischen Meinungen auf beiden Seiten des Status quo teilte Joseph P. Overton in Stufen auf. Im Zentrum des Fensters und als Abbild des Ist- 
Zustands finden sich die populären Positionen. Dann kommen die sinnvollen, dann die gerade noch akzeptablen. Außerhalb des Fensters finden sich die radikalen und noch weiter außen die undenkbaren Positionen. Das Fenster selbst umfasst nur die enge Mitte dieses Spektrums, die einigermaßen populären Positionen links und rechts vom Status quo.

Weil nun in der Demokratie Politiker nur Dinge vertreten und umsetzen, die im Kern von Gesellschaft und Öffentlichkeit als akzeptabel betrachtet werden, nutzen Gegner der Demokratie das Konzept in diametraler Absicht: Denn sie wissen, dass sie zuerst die öffentliche Meinung verschieben müssen, bevor sich die Politik ändert. Sie tun das mit zwei Instrumenten. Einerseits mit »Fake News « und Hate Speech, die in den Echokammern der sozialen Netzwerke mit Resonanz aufgeladen werden, andererseits mit dem systematischen Bestreiten der Wahrheitsfunktion gemeinwohlorientierter Kommunikation, vulgo: Journalismus. In perfider Weise kommt jedoch in jüngster Zeit hinzu, dass die journalistischen Objektivitätsbestrebungen im postfaktischen Zeitalter gegen den Journalismus gewendet werden: Jay Rosen (2010) hat das (falschverstandene) Streben nach Neutralität und Objektivität in Anlehnung an den US-amerikanischen Philosophen Thomas Nagel als »View from Nowhere « (Rosen, 2010) bezeichnet und kritisiert die journalistische Standpunktlosigkeit als falsch verstandene Neutralitätsillusion. Diese korrespondiert stark mit dem deutschen Pendant dieser Standpunktlosigkeit, für die meist Hanns Joachim Friedrichs‘ Journalismus-Mantra, »einen guten Journalisten erkennt man daran, daß er Distanz zum Gegenstand seiner Betrachtung hält; daß er sich nicht gemein macht mit einer Sache, auch nicht mit einer guten Sache, daß er immer dabei ist, aber nie dazugehört« (Friedrichs, 1994), in den Zeugenstand gerufen wird.

Unbesehen der Tatsache, dass Friedrichs (1994) sowohl im Klappentext seiner Autobiographie »Journalistenleben« als auch im berühmten SpiegelInterview kurz vor seinem Tod ein Statement zur Tatsachenwahrheit abgegeben hatte und nur den Kern des Nachrichtenjournalismus, nicht aber die Haltung zur demokratischen Weltanschauung meinte, werden diese scheinbaren Belege für das Neutralitätsgebot unterdessen in populistischer Weise instrumentalisiert. Es heißt dann, die überwiegend linksliberale Presse sei vom Geist des Gutmenschentums beseelt, mache sich mit multikulturellen Wertvorstellungen gemein, lobe die Willkommenskultur, unterstütze aktiv und einseitig die Migration nach Deutschland und habe die Grundprinzipien des neutralen Informationsjournalismus aufgegeben.

So beklagte Sandro Schroeder (2017) im Anschluss an die Debatte, ob sich deutsche Journalistinnen und Journalisten im Jahr 2017 in legitimer Weise an einem politischen Aufruf zur Befreiung des in der Türkei inhaf- 
tierten Kollegen Deniz Yücel beteiligen durften, dass im metajournalistischen Diskus um so genannte Systemmedien, Mainstream-Medien oder die böse Lügenpresse gerne das Friedrichs-Zitat als Totschlagsargument hervorgeholt wurde, um Journalisten zu gängeln: »Ihnen [den Kritikern] geht es unter dem Vorwurf des Nicht-gemein-Machens darum, dass JournalistInnen auch extreme Positionen als gleichwertig darstellen - eine falsche Vorstellung von journalistischer Neutralität. Es ist die ,Das wird man ja wohl noch sagen dürfen'-Vorstellung von Meinungsfreiheit, die auch bitteschön Falsches und Menschenverachtendes zu tolerieren habe. Mit dem klaren Ziel, das Overton-Fenster für ihre Zwecke zu verschieben, um Radikales zu normalisieren « (Schroeder, 2017). Die Folge ist, dass die professionelle Berufsnorm des Neutralitätsgebots und eine daraus resultierende Standpunktlosigkeit umgedeutet werden in eine Aufforderung zur Toleranz gegenüber Lügen, Unwahrheiten und Behauptungen, die sich gegen eine offene Gesellschaft richten und zur Akzeptanz von Positionen, mit denen die Abschaffung demokratischer Institutionen vorbereitet wird. Aus dem Umfeld der AfD wird mit Bezug auf die kritische Berichterstattung zu extremistischen Bewegungen wie PEGIDA, dem Flügel und den Identitären immer wieder die Kritik geäußert, die klassischen Nachrichtenmedien verfehlten die Qualitätsnormen Ausgewogenheit, Unparteilichkeit, Unabhängigkeit und Neutralität.

Die Post-Truth-Ära stresst damit den Journalismus. Wie kann und muss er sich behaupten, jetzt da er nicht nur wirtschaftlich und publizistisch, sondern auch in Bezug auf Debatten- und Diskurshoheit seine Meinungsführerschaft verloren hat und das Leitmedienprinzip in der öffentlichen Kommunikation kein Monopol mehr besitzt? Es würde in dieser Situation schon helfen, wenn die Kommunikationswissenschaft laut und vernehmlich erklärt, dass die Qualitätsnorm der Ausgewogenheit sich nur auf den Bezugsrahmen des Gesellschaftssystems und der in ihm konsentierten Werte bezieht, dass das Overton-Fenster allein die pluralistische, offene Gesellschaft, den Rechtsstaat und die freiheitlich-demokratische Grundordnung umspannt. Und wenn klar artikuliert wird, dass das Ausgewogenheitspostulat nur für das zentrale Meinungsspektrum von Demokratie und für den Kernbereich der pluralistischen Gesellschaft gilt, nicht aber für rechtsextremistische Lügenpressevorwürfe, xenophobe Verschwörungstheorien, »Fake News" und Alternative Fakten. Es gilt nicht für so genannte Meinungsangebote, die sich außerhalb des Wertekanons befinden und welche Staatsform, Gesellschaftssystem und die kulturellen und gesellschaftspolitischen Übereinkünfte und Identitäten eines Landes angreifen. Evelyn Roll (2016) glaubt, der Begriff Post-Truth verschleiere die Wirklichkeit, weil er 
ein evolutionäres Prinzip unterstellt, das zwangsläufig und nicht abzuändern sei: »Wer dann noch ,Zeitalter' dazu schreibt, ,postfaktisches Zeitalter', hat die Demokratie schon aufgegeben. Er unterwirft sich der fixen Idee, es sei an dieser sich selbst erzeugenden und verstärkenden Lügenkultur, vor allem in den sogenannten sozialen Medien, nun einmal nichts mehr zu ändern « (Roll, 2016). Wenn man dem Gedanken folgt, dass das Zeitalter der subjektiven Wahrheiten und Alternativen Fakten einer politischen Logik der Dialektik folgt, deren Pendelschwung sich gerade von freiheitlich-liberal nach autoritär-restaurativ in Bewegung gesetzt hat, dann ist die Gewissheit nah, dass das Pendel auch wieder zurückschwingen wird. Es wird umso schneller zurückschwingen, je besser es Politik, Recht, Medien und Wissenschaft gelingt, ihre kognitiven Institutionen - also die als absolut und überzeitlich geltenden Regeln und Normen, die das System erhalten - zu schützen. Und wie? Indem man Lügen als Lügen entlarvt, Fakten als Fakten evident macht und Bürgerinnen und Bürger, Wählerinnen und Wähler, Rezipientinnen und Rezipienten dazu befähigt, einen Unterschied zu machen, der einen Unterschied macht. Oder um mit Deichkind zu sprechen: »Dummheit ist nicht erblich«.

\section{Post Scriptum}

In den ersten Monaten der weltweiten Corona-Pandemie im Frühjahr 2020 hatte es zunächst den Anschein, dass faktenbasierte Kommunikation und ein evidenzorientierter Wissenschaftsjournalismus in der Öffentlichkeit ein unverhofftes Comeback feiern würden und die öffentliche Meinung von den biowissenschaftlichen Erkenntnissen und Befunden dominiert sei, die Virologie und Epidemiologie liefern konnten. Dieser Zustand einer gewissen Wissenschaftsrenaissance hielt in Deutschland ab dem Zeitpunkt der politisch verordneten Schulschließungen und Ausgangsbeschränkungen für genau vier Wochen an. Dann erlangten Verschwörungsmythen, Kampagnen und eine forsche Lügenkultur erneut in den sozialen Netzwerken die Diskurshoheit. 


\section{Literatur}

Arendt, Hannah (2013). Wahrheit und Lüge in der Politik. Zwei Essays. München: Piper.

Breuer, Ingeborg (2016). Postfaktisch. Wort des Jahres 2016. Die gefühlte Wahrheit. Deutschlandfunk, 15. Dezember 2016. Abgerufen von https://www.deutschland funk.de/die-gefuehlte-wahrheit-postfaktisch-wort-des-jahres-

2016.1148.de.html?dram:article_id=373840. Abgerufen am 01. Juli 2020.

Chess, Shira \& Adrienne Shaw (2015). A Conspiracy of Fishes, or, How We Learned to Stop Worrying About \#GamerGate and Embrace Hegemonic Masculinity. Journal of Broadcasting \& Electronic Media, 59(1), 208-220. DOI: 10.1080/08838151.2014.9 99917.

Friedrichs, Hanns Joachim (1994). Journalistenleben. München: Droemer Knaur.

Haaf, Meredith (2020). Die Wahrheit ist unbequem. Meinung versus Fakten. Süddeutsche Zeitung, 26. Januar 2020, 4.

Haberkorn, Tobias (2018). Fake News sind immer die anderen. »Alternative Fakten«. zeit.de, 16. Januar 2018. Abgerufen von https://www.zeit.de/kultur/2018-01/ alternative-fakten-unwort-des-jahres-donald-trump. Abgerufen am 01. Juli 2020.

Habermas, Jürgen (1981). Theorie des kommunikativen Handelns. Frankfurt am Main: Suhrkamp.

Hendricks, Vincent F. \& Mads Vestergaard (2018). Postfaktisch. Die neue Wirklichkeit in Zeiten von Bullshit, Fake News und Verschwörungstheorien. München: Blessing Verlag.

Jacobsen, Lenz (2018). Krasse Meinungen wehen uns mit voller Wucht ins Gesicht. zeit.de, 26. Juli 2018. Abgerufen von https:/www.zeit.de/politik/ deutschland/2018-07/overtone-fenster-diskussionen-debatten-diskurse-radikal. Abgerufen am 18. März 2020.

Keyes, Ralph (2004). The Post-Truth Era. Dishonesty and Deception in Contemporary Life. New York: St. Martin's Publishing Group.

Kummer, Tom (2007). Blow up. Eine wahre Geschichte im Zeitalter des Fakes. München: Blumenbar Verlag.

Lehmann, Armin (2020). »Eine Frage des Überlebens für den ganzen Kontinent«. Kanzlerin Merkel in Davos. Der Tagesspiegel, 23. Januar 2020. Abgerufen von https://www.tagesspiegel.de/politik/kanzlerin-merkel-in-davos-eine-frage-desueberlebens-fuer-den-ganzen-kontinent/25464246.html. Abgerufen am 01. Juli 2020.

Popper, Raimund (1989). Logik der Forschung. Zur Erkenntnistheorie der modernen Naturwissenschaft. Tübingen: Verlag J. C. B. Mohr.

Roll, Evelyn (2016). Die Lüge. Demokratie versus postfaktisches Zeitalter. sueddeutsche.de, 18. November 2016. Abgerufen von https:/www.sueddeutsche.de/leben/ demokratie-vs-postfaktisches-zeitalter-die-luege-1.3254089. Abgerufen am 18. März 2020.

Rosen, Jay (2010). The View from Nowhere. Questions and Answers. Pressthink, 10. November 2010. Abgerufen von https://pressthink.org/2010/11/the-view-fromnowhere-questions-and-answers/. Abgerufen am 18. März 2020. 
Schroeder, Sandro (2017). »Journalisten machen sich mit keiner Sache gemein, auch nicht mit einer guten«. Sandro Schroeder Blog, 5. März 2017. Abgerufen von https://sandro-schroeder.de/journalisten-selbstverstaendnis/. Abgerufen am 18. März 2020.

Schwan, Gesine (2017). Verständigung in Zeiten des »Postfaktischen«. In: Strasser, J. (Hrsg.), Das freie Wort. Vom öffentlichen Gebrauch der Vernunft im postfaktischen Zeitalter (41-54). München: Allitera Verlag.

Siegmund, Arne (2019). Deichkind veröffentlichen großartiges »Tagesschau«-Video zur neuen Single. Watson, 26. Juni 2019. Abgerufen von https://www.watson.de/ unterhaltung/musik/671344013-tagesschau-so-grossartig-manipulieren-deichkindclips-fuer-ihren-neuen-song. Abgerufen am 18. März 2020.

Strasser, Johano (2017). Zur Einleitung. In: Strasser, J. (Hrsg.), Das freie Wort. Vom öffentlichen Gebrauch der Vernunft im postfaktischen Zeitalter (7-30). München: Allitera Verlag.

Tesich, Steve (1992). A Government of Lies. The Nation. Januar 6/13, 12-14.

Weber, Stefan (2002). Was heißt »Medien konstruieren Wirklichkeit «? Von einem ontologischen $\mathrm{zu}$ einem empirischen Verständnis von Konstruktion. Medienimpulse, 40, 11-16. 